\title{
Preoperative coronary artery calcifications in veterans predict higher all-cause mortality in early-stage lung cancer: a cohort study
}

\author{
Maren E. Shipe ${ }^{1}$, Amelia W. Maiga ${ }^{1,2}$, Stephen A. Deppen ${ }^{1,2}$, Gretchen C. Edwards ${ }^{1,2}$, \\ Hannah N. Marmor ${ }^{1}$, Rhonda Pinkerman ${ }^{2}$, Gary T. Smith ${ }^{3,4}$, Elizabeth Lio ${ }^{3}$, Johnny L. Wright ${ }^{3}$, \\ Chirayu Shah $^{3,4}$, Jonathan C. Nesbitt ${ }^{1,2}$, Eric L. Grogan ${ }^{1,2}$ \\ ${ }^{1}$ Department of Thoracic Surgery, Vanderbilt University Medical Center, Nashville, TN, USA; ${ }^{2}$ Department of Surgery, Tennessee Valley Healthcare \\ System, Nashville, TN, USA; ${ }^{3}$ Department of Radiology, Vanderbilt University Medical Center, Nashville, TN, USA; ${ }^{4}$ Department of Radiology, \\ Tennessee Valley Healthcare System, Nashville, TN, USA \\ Contributions: (I) Conception and design: ME Shipe, AW Maiga, SA Deppen, EL Grogan; (II) Administrative support: None; (III) Provision of \\ study materials or patients: R Pinkerman, E Lio, GT Smith, JL Wright, C Shah; (IV) Collection and assembly of data: ME Shipe, AW Maiga, GC \\ Edwards; (V) Data analysis and interpretation: ME Shipe, AW Maiga, SA Deppen, EL Grogan; (VI) Manuscript writing: All authors; (VII) Final \\ approval of manuscript: All authors. \\ Correspondence to: Eric L. Grogan, MD, MPH, FACS. Department of Thoracic Surgery, 609 Oxford House, 131321 st Ave. South, Nashville, TN \\ 37232, USA. Email: eric.grogan@vumc.org.
}

\begin{abstract}
Background: Lung cancer patients often have comorbidities that may impact survival. This observational cohort study examines whether coronary artery calcifications (CAC) impact all-cause mortality in patients with resected stage I non-small cell lung cancer (NSCLC).

Methods: Veterans with stage I NSCLC who underwent resection at a single institution between 2005 and 2018 were selected from a prospectively collected database. Radiologists blinded to patient outcomes graded CAC severity (mild, moderate, or severe) in preoperative CT scans using a visual estimation scoring system. Inter-rater reliability was calculated using the kappa statistic. All-cause mortality was the primary outcome. Kaplan-Meier survival analysis and Cox proportional hazards regression were used to compare time-to-death by varying CAC.

Results: The Veteran patients ( $\mathrm{n}=195)$ were predominantly older (median age of 67$)$ male (98\%) smokers (96\%). The majority (68\%) were pathologic stage IA. Overall, $12 \%$ of patients had no CAC, $27 \%$ mild, $26 \%$ moderate, and $36 \%$ severe CAC. Median unadjusted survival was 8.8 years for patients with absent or mild CAC versus 6.3 years for moderate and 5.9 years for severe $\mathrm{CAC}(\mathrm{P}=0.01)$. The adjusted hazard ratio for moderate CAC was 1.44 (95\% CI, 0.85-2.46) and for severe CAC was 1.73 (95\% CI, 1.03-2.88; P for trend <0.05).

Conclusions: The presence of severe CAC on preoperative imaging significantly impacted the all-cause survival of patients undergoing resection for stage I NSCLC. This impact on mortality should be taken into consideration by multidisciplinary teams when making treatment plans for patients with early-stage disease.
\end{abstract}

Keywords: Lung cancer; coronary artery calcifications (CAC); mortality; early stage

Submitted Jun 02, 2020. Accepted for publication Dec 25, 2020.

doi: $10.21037 /$ jtd-20-2102

View this article at: http://dx.doi.org/10.21037/jtd-20-2102

\section{Introduction}

Coronary artery calcifications (CAC), a radiographic sign of cardiovascular disease (CVD), are well established as a robust predictor of all-cause mortality in smokers and non-smokers alike (1-8). This is true above and beyond traditional cardiovascular risk factors included in the Framingham Coronary Heart Disease Risk Score and the American College of Cardiology/American Heart 
Association Pooled Cohort Equations such as smoking, cholesterol levels, and blood pressure (6). In one study, among patients without any traditional risk factors (current cigarette smoking, dyslipidemia, diabetes, hypertension, family history of coronary heart disease), increasing CAC scores were associated with a 3.00 to 13.38 -fold higher allcause mortality risk when adjusted for age and sex. Of note, patients without any risk factors but with a high CAC score $(\geq 400)$ had a significantly higher all-cause mortality rate than patients with multiple risk factors but no CAC (9).

More recently, CAC scores have also been shown to be independent predictors of all-cause mortality and of fatal and non-fatal cardiac events in several lung cancer screening populations, including the National Lung Screening Trial (NLST) and the Danish Lung Cancer Screening Trial (10-15). As many as half of patients undergoing lung cancer screening had CAC incidentally found on computed tomography (CT) scans, while fewer than one-third of these patients carried a pre-screening diagnosis of CAD $(16,17)$. In the NLST population, $50 \%$ of deaths were related to cardiovascular events, and CAC identified on screening CT scans were a very high predictor of major cardiovascular events and mortality even without a prior diagnosis of $\operatorname{CAD}(13,18,19)$. The lung cancer screening population is of interest because it constitutes an enriched population of current and former smokers in which there is not only the mortality benefit of identifying early-stage lung cancer, but also a highly teachable moment to intervene on smoking cessation and secondary and tertiary CVD prevention alike (20-22).

Comparatively little attention has been paid towards assessing CAC in patients diagnosed with lung cancer, despite the fact that this population of predominantly older current and former heavy smokers is at least at intermediate risk of coronary artery disease (CAD) $(10,11,15)$. Predicting major cardiovascular events after lung resection is of critical importance $(23,24)$. The prevalence of CAC in patients with early-stage lung cancer is just beginning to be explored; one small study found almost $90 \%$ of patients with lung cancer who were screened for CAC were found to have plaques (25). Similar to results from screening studies, the majority of lung cancer patients identified to have CAC did not carry a preexisting diagnosis of CAD, CVD, or peripheral artery disease (PAD) (26). This is concerning as patients with a preoperative diagnosis of $\mathrm{CAD}$ have increased mortality following open and minimally invasive lung resections (27-29). However, the direct impact of CAC on mortality in lung cancer patients has yet to be determined. The primary objective of our study was to investigate whether preoperative CAC independently increases all-cause mortality in patients with resected pathologic stage I non-small cell lung cancer (NSCLC).

We present the following article in accordance with the STROBE reporting checklist (available at http://dx.doi. org/10.21037/jtd-20-2102).

\section{Methods}

\section{Study population}

We used a single-institution cohort study design, which was comprised of Veterans who underwent surgical resection for pathologic stage I NSCLC at the Tennessee Valley Healthcare Center (TVHS) Veterans Hospital, a tertiary Veterans Affairs (VA) facility in Nashville, TN, over the 13-year period from 2005 to 2018. Patient demographics and clinical covariates were collected from chart review. There was no missing data from study participants. The study was conducted in accordance with the Declaration of Helsinki (as revised in 2013). The study was approved by the TVHS Institutional Review Board (registration number 00005465) and individual consent for this retrospective analysis was waived.

Eligibility criteria included patients who underwent a lung resection (open or minimally invasive) at TVHS during the above dates and had a final pathologic diagnosis of stage I NSCLC. Exclusion criteria included a final diagnosis of benign disease and the absence of readable computed tomography or positron-emission tomography (PET)/CT images in the VA medical record. For patients who underwent a second lung resection for a separate nodule or for a known or suspected recurrence during the study period, only the first surgical resection was included, and time-to-death was calculated from the initial resection.

\section{Outcome}

The primary outcome was all-cause mortality as determined by chart review. Cause-specific mortality data was limited only to deaths which occurred in the Veterans Hospital, representing a skewed population. As cause-specific mortality was available for only a small percentage of the study population, all-cause mortality was selected as our primary outcome. Secondary outcomes included years of follow up and time to death. Years of follow up was determined by date of last contact with the Veteran. Loss to follow up was censored from our survival analysis. 
Table 1 Patient characteristics by grade of CAC

\begin{tabular}{|c|c|c|c|c|c|}
\hline Characteristics & Total population $(n=195)$ & Absent/mild CAC $(n=75)$ & Moderate CAC $(n=50)$ & Severe CAC $(n=70)$ & $P$ value \\
\hline Male gender & $192(98 \%)$ & $72(97 \%)$ & 49 (98\%) & $70(100 \%)$ & 0.4 \\
\hline Smokers & $189(96 \%)$ & $71(95 \%)$ & $48(96 \%)$ & $70(100 \%)$ & 0.1 \\
\hline Median pack-years (IQR) & $60(40,99)$ & $60(40,97)$ & $60(40,99)$ & $55(40,97)$ & 0.31 \\
\hline Pathological & & & & & 0.6 \\
\hline Stage IA & $132(68 \%)$ & $48(64 \%)$ & $34(68 \%)$ & $50(71 \%)$ & \\
\hline Stage IB & $63(32 \%)$ & 27 (36\%) & $16(32 \%)$ & $20(29 \%)$ & \\
\hline
\end{tabular}

CAC, coronary artery calcification; IQR, interquartile range; BMI, body mass index $\left(\mathrm{kg} / \mathrm{m}^{2}\right)$.

\section{Exposure}

The exposure of interest was the presence and degree of preoperative CAC. Preoperative chest CT or PET/ CT images were reviewed separately by two radiologists, with a third radiologist serving as a tiebreaker if needed. Radiologists identified the presence and grade (mild, moderate, or severe) of CAC as determined using the visual estimation method (13). CAC was considered present if any coronary calcium was visualized in the right, left main, left anterior descending, or left circumflex distribution. CAC was considered mild if less than $5 \%$ of the total length of the coronary arteries were calcified, moderate if $5-20 \%$, and severe if greater than $20 \%$ by visual estimate.

This study used the visual estimation method for grading CAC on CT scans as described above. CAC are often formally calculated using the semi-automated Agatston coronary calcium score, which requires a specific quality CT scan as well as specialized software (30). However, multiple previous reports demonstrate that visual scoring of CAC on CT correlates well with semi-automated Agatston scoring and is predictive of all-cause and CVD mortality $(13,15,31,32)$. Additionally, visual estimation of CAC was recently reaffirmed as adequate for the lung cancer screening population in a joint consensus report from the Society of Cardiovascular Computed Tomography and the Society of Thoracic Radiology (8).

\section{Statistical analysis}

R Studio software version 0.99 .896 were used for all statistical analyses. Patient demographics and clinical characteristics were summarized using descriptive statistics.
Kaplan-Meier survival analysis and Cox proportional hazards regression were used to compare time-to-death by varying $\mathrm{CAC}$ after adjusting for patient age and pathologic stage.

\section{Results}

Table 1 describes the study population. A total of 195 veterans were found eligible for our study using criteria as mentioned above. This determined our sample size. As expected in this Veteran cohort, patients were predominantly older male smokers, with a median packyear history of 60 [interquartile range (IQR), 40-90]. 68\% of patients had stage IA NSCLC while $32 \%$ had stage IB NSCLC. The stage distribution did not significantly differ by CAC group. Overall, 23 Veterans (12\%) had no CAC, 52 (27\%) had mild CAC, 50 (26\%) had moderate CAC, and 70 $(36 \%)$ had severe CAC. The median time between CT scan and surgery was 56 days. Inter-rater reliability between two radiologists for grade of CAC was $80 \%$.

The median follow-up time was 3.5 years; this significantly differed by CAC grade with those with severe CAC having a shorter median follow-up time (3.1 vs. 4.8 years for those with absent or mild CAC.) Table 2 demonstrates that overall, $50 \%$ of patients died during the study period and $22 \%$ within 2 years of surgery. Survival at 2 years was $87 \%$ for absent/mild CAC, $76 \%$ for moderate CAC, and $71 \%$ for severe CAC, which was not significantly different between groups.

Figure 1 shows the Kaplan-Meier curve for survival by grade of CAC. Median unadjusted survival was 8.8 years for patients with absent or mild CAC, versus 6.3 years for 
Table 2 Patient outcomes by grade of CAC

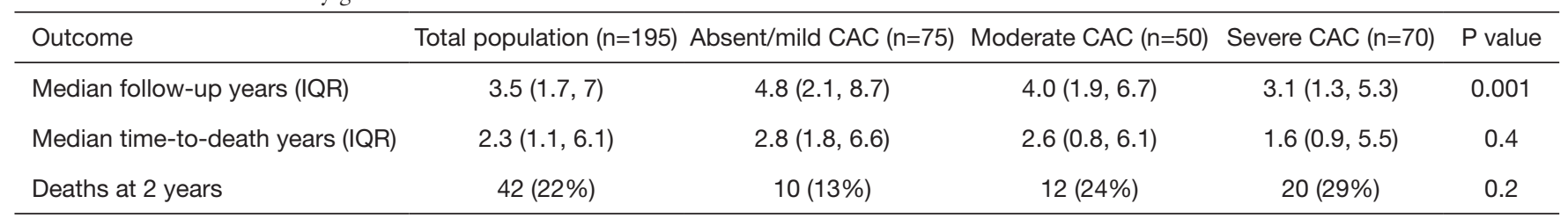

CAC, coronary artery calcification; IQR, interquartile range.

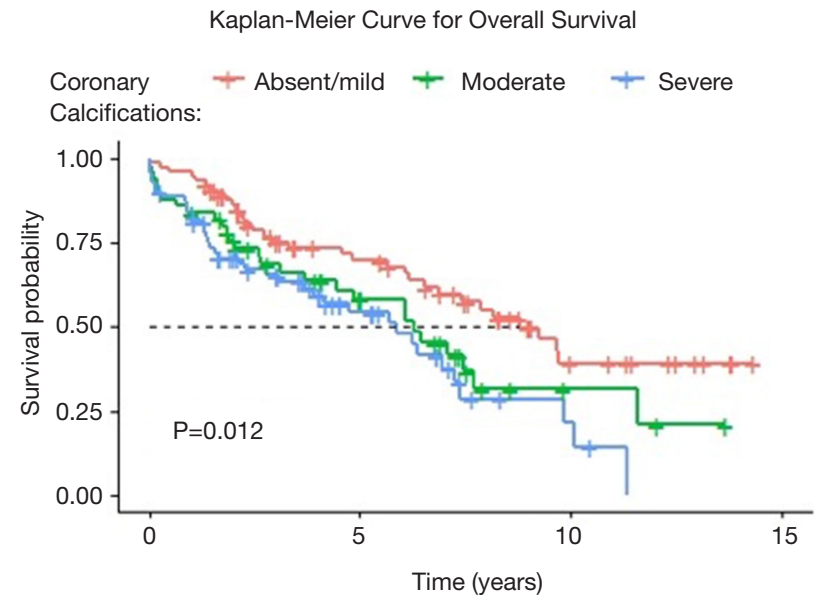

Figure 1 Kaplan-Meier curve for overall survival by grade of CAC. CAC, coronary artery calcification.

moderate $\mathrm{CAC}$ and 5.9 years for severe $\mathrm{CAC}(\mathrm{P}=0.01)$.

The results of the multivariate Cox proportional hazards analysis adjusted for age and pathologic stage are presented in Table 3. As compared to patients with absent/mild CAC, the hazard ratio for patients with moderate CAC was 1.44 (95\% CI, 0.85-2.46) and for severe CAC was 1.73 (95\% CI, $1.03-2.88$; P for trend $<0.05$ ).

\section{Discussion}

Patients diagnosed with lung cancer have high-risk comorbidities that can impact overall survival. CAC is a known risk factor for major cardiac events and death in surgical patients, regardless of prior diagnosis of CAD or CVD (1-8). While studies have analyzed the impact of CAC on mortality in lung cancer screening patients, the impact on surgically resectable lung cancer patients has not been determined (10-15). This study evaluated the impact of CAC identified on preoperative CT scans on all-cause mortality in stage I NSCLC surgical patients. We found that patients with severe CAC were significantly more likely
Table 3 Results of multivariate Cox proportional hazard analysis

\begin{tabular}{lcc}
\hline Covariate & Hazard ratio $(95 \% \mathrm{Cl})$ & P value \\
\hline Age (per year) & $1.02(1.0-1.05)$ & 0.09 \\
Stage & Ref & 0.8 \\
IA & $1.04(0.68-1.60)$ & \\
IB & Ref & 0.03 \\
CAC & \\
Absent/mild & $1.44(0.85-2.46)$ & \\
Moderate & $1.73(1.03-2.88)$ \\
Severe &
\end{tabular}

to die than patients with absent or mild CAC, even when controlling for age of the patient and stage of NSCLC. Patients with severe CAC survived nearly 2 years less than patients with mild or absent CAC (median of 5.9 years after surgery) and were 1.7 times more likely to die within the study period.

In light of these findings, it would be unwise for surgeons and other clinicians to disregard an incidental finding of CAC in lung cancer patients. As of 2016, the American College of Radiology mandates the reporting of moderate or severe CAC in their National Lung Cancer Screening Registry (8). In a population similar to ours, a large proportion of patients (62\% of our patients had moderate/ severe CAC) may present with this finding. In terms of generalizability, our study population exhibited typical characteristics of a Veteran cohort; older, male smokers.

Additionally, the diagnosis of cancer has been shown to be associated with the development of CAC even after taking traditional CVD risk factors into account, particularly in women $(33,34)$.

Unfortunately, CAC grade is not currently incorporated into common preoperative risk assessment scales. Current practice prior to lung resection includes calculation of 
the thoracic revised cardiac risk index (ThRCRI), which predicts absolute and relative cardiovascular risk after major lung resection, and gives 1.5 points out of a total 5.5 points for a history of CAD or congestive heart failure but does not include CAC presence or grade (35). Similarly, the Veterans Affairs Surgical Quality Improvement Program (VASQIP) risk calculator, used before all VA surgeries, does not include the presence or degree of CAC in its assessment (36). Thoracic surgeons and multidisciplinary teams should work to include CAC in a systematic way in risk stratification or as part of the shared decision-making process in NSCLC, as CAC can significantly impact mortality even in earlystage, resectable lung cancers and could alter patients' goals of care and treatment plans.

Limitations of our study include its single-center design and small sample size $(n=195)$, which limited the ability to perform an analysis adjusting for smoking exposure, pulmonary function, R0/R1 surgical resection, other variables in the models, as well as other risk factors for death among lung cancer patients. This limited adjustment analysis may falsely relate CAC to mortality. However, previous studies, including the NELSON trial in lung cancer screening participants, have established the predictive contribution of CAC even after adjusting for smoking pack-years (14). Because of a lack of cause-specific mortality data, our outcome was all-cause mortality, and thus an unknown proportion of deaths may have been due to cancer or causes other than CAD. Finally, the quality of chest CT imaging varied by patient, and radiologic studies were not originally performed for the purpose of evaluating CAC, which limits our ability to accurately detect and grade CAC. However, multiple recent studies have demonstrated that $\mathrm{CT}$ scans done for other indications can be adequate for assessment of CAC, in particular low-dose screening CT scans for lung cancer $(37,38)$.

\section{Conclusions}

In conclusion, the presence of severe CAC on preoperative imaging significantly impacted the all-cause survival of patients undergoing resection for stage I NSCLC. This impact on mortality should be taken into consideration by multidisciplinary teams when making treatment plans for patients with early-stage disease. Further work is needed to determine how the presence and degree of CAC impacts more diverse populations and in other stages of NSLC, and how to incorporate this information into preoperative shared decision-making discussions with patients.

\section{Acknowledgments}

Dr. Grogan is a former recipient of the Department of Veterans Affairs, Veterans Health Administration, Health Services Research and Development Service Career Development Award (10-024). The views expressed in this article are those of the authors and do not necessarily represent the views of the Department of Veterans Affairs. The funding agency had no role in the design and conduct of the study; collection, management, analysis, and interpretation of the data; the preparation, review, or approval of the manuscript; or in the decision to submit the manuscript for publication.

Funding: Dr. Shipe was supported by the Agency for Healthcare Research (AHRQ) under Award Number T32 HS026122. Drs. Maiga and Edwards were supported by the Office of Academic Affiliations, Department of Veterans Affairs (VA) National Quality Scholars Program.

\section{Footnote}

Reporting Checklist: The authors have completed the STROBE reporting checklist. Available at http://dx.doi. org/10.21037/jtd-20-2102

Data Sharing Statement: Available at http://dx.doi. org/10.21037/jtd-20-2102

Conflicts of Interest: All authors have completed the ICMJE uniform disclosure form (available at http://dx.doi. org/10.21037/jtd-20-2102). The authors have no conflicts of interest to declare.

Ethical Statement: The authors are accountable for all aspects of the work in ensuring that questions related to the accuracy or integrity of any part of the work are appropriately investigated and resolved. The study was conducted in accordance with the Declaration of Helsinki (as revised in 2013). The study was approved by the TVHS Institutional Review Board (registration number 00005465) and individual consent for this retrospective analysis was waived.

Open Access Statement: This is an Open Access article distributed in accordance with the Creative Commons Attribution-NonCommercial-NoDerivs 4.0 International License (CC BY-NC-ND 4.0), which permits the noncommercial replication and distribution of the article with 
the strict proviso that no changes or edits are made and the original work is properly cited (including links to both the formal publication through the relevant DOI and the license). See: https://creativecommons.org/licenses/by-nc-nd/4.0/.

\section{References}

1. Madhavan MV, Tarigopula M, Mintz GS, et al. Coronary artery calcification: Pathogenesis and prognostic implications. J Am Coll Cardiol 2014;63:1703-14.

2. Howard G, Wagenknecht LE, Burke GL, et al. Cigarette smoking and progression of atherosclerosis: The atherosclerosis risk in communities (ARIC) study. JAMA 1998;279:119-24.

3. Shaw LJ, Raggi P, Callister TQ, et al. Prognostic value of coronary artery calcium screening in asymptomatic smokers and non-smokers. Eur Heart J 2006;27:968-75.

4. McEvoy JW, Blaha MJ, Rivera JJ, et al. Mortality rates in smokers and nonsmokers in the presence or absence of coronary artery calcification. JACC Cardiovasc Imaging 2012;5:1037-45. Erratum in: JACC Cardiovasc Imaging 2013;6:747.

5. Schulman-Marcus J, Valenti V, Hartaigh BÓ, et al. Prognostic utility of coronary artery calcium scoring in active smokers: A 15-year follow-up study. Int J Cardiol 2014;177:581-3.

6. Stone NJ, Robinson JG, Lichtenstein AH, et al. 2013 ACC/AHA Guideline on the Treatment of Blood Cholesterol to Reduce Atherosclerotic Cardiovascular Risk in Adults. J Am Coll Cardiol 2014;63:2889-2934.

7. Grundy SM, Cleeman JI, Merz CNB, et al. Implications of recent clinical trials for the National Cholesterol Education Program Adult Treatment Panel III Guidelines. J Am Coll Cardiol 2004;44:720-32.

8. Hecht HS, Cronin P, Blaha MJ, et al. 2016 SCCT/ STR guidelines for coronary artery calcium scoring of noncontrast noncardiac chest CT scans: A report of the Society of Cardiovascular Computed Tomography and Society of Thoracic Radiology. J Cardiovasc Comput Tomogr 2017;11:74-84.

9. Nasir K, Rubin J, Blaha MJ, et al. Interplay of coronary artery calcification and traditional risk factors for the prediction of all-cause mortality in asymptomatic individuals. Circ Cardiovasc Imaging 2012;5:467-73.

10. Rasmussen T, Kober L, Abdulla J, et al. Coronary artery calcification detected in lung cancer screening predicts cardiovascular death. Scand Cardiovasc J 2015;49:159-67.

11. Vehmas T. Visually scored calcifications in thoracic arteries predict death: Follow-up study after lung cancer CT screening. Acta radiol 2012;53:643-7.

12. Jacobs PC, Gondrie MJA, Van Der Graaf Y, et al. Coronary artery calcium can predict all-cause mortality and cardiovascular events on low-dose ct screening for lung cancer. AJR Am J Roentgenol 2012;198:505-11.

13. Chiles C, Duan F, Gladish GW, et al. Association of coronary artery calcification and mortality in the national lung screening trial: A comparison of three scoring methods. Radiology 2015;276:82-90.

14. Takx RAP, Išgum I, Willemink MJ, et al. Quantification of coronary artery calcium in nongated CT to predict cardiovascular events in male lung cancer screening participants: Results of the NELSON study. J Cardiovasc Comput Tomogr 2015;9:50-7.

15. Watts JR, Sonavane SK, Snell-Bergeon J, et al. Visual scoring of coronary artery calcification in lung cancer screening computed tomography: Association with allcause and cardiovascular mortality risk. Coron Artery Dis 2015;26:157-62.

16. Morgan L, Choi H, Reid M, et al. Frequency of incidental findings and subsequent evaluation in low-dose computed tomographic scans for lung cancer screening. Ann Am Thorac Soc 2017;14:1450-6.

17. Mendoza DP, Kako B, Digumarthy SR, et al. Impact of Significant Coronary Artery Calcification Reported on Low-Dose Computed Tomography Lung Cancer Screening. J Thorac Imaging 2020;35:129-35.

18. Fan L, Fan K. Lung cancer screening CT-based coronary artery calcification in predicting cardiovascular events: A systematic review and meta-analysis. Medicine (Baltimore) 2018;97:e10461.

19. Malcolm KB, Dinwoodey DL, Cundiff MC, et al. Qualitative coronary artery calcium assessment on CT lung screening exam helps predict first cardiac events. J Thorac Dis 2018;10:2740-51.

20. National Lung Screening Trial Research Team, Aberle D, Adams A, et al. Reduced Lung-Cancer Mortality with Low-Dose Computed Tomographic Screening. N Engl J Med 2011;365:395-409.

21. Tammemägi MC, Berg CD, Riley TL, et al. Impact of lung cancer screening results on smoking cessation. J Natl Cancer Inst 2014;106:dju084.

22. Park ER, Gareen IF, Japuntich S, et al. Primary care provider-delivered smoking cessation interventions and smoking cessation among participants in the national lung screening trial. JAMA Intern Med 2015;175:1509-16.

23. Gaissert HA, Fernandez FG, Allen MS, et al. The 
Society of Thoracic Surgeons General Thoracic Surgery Database: 2016 Update on Research. Ann Thorac Surg 2016;102:1444-51.

24. Rivera MP, Tanner NT, Silvestri GA, et al. Incorporating coexisting chronic illness into decisions about patient selection for lung cancer screening an official American thoracic society research statement. Am J Respir Crit Care Med 2018;198:e3-13.

25. Zhang F, Chen J, Yang ZG, et al. Preoperative Assessment of Coronary Arteries by Cardiac Computed Tomography in Patients with Lung Cancer. Thorac Cardiovasc Surg 2020;68:169-75.

26. Cuddy S, Payne S, Murphy D, et al. Prevalence of coronary artery calcification in patients with early stage lung cancer and missed opportunities for cardiovascular risk optimization. BMJ 2017. doi: 10.1136/heartjnl-2017ics17.52.

27. Sandri A, Petersen RH, Decaluwé H, et al. Coronary artery disease is associated with an increased mortality rate following video-assisted thoracoscopic lobectomy. J Thorac Cardiovasc Surg 2017;154:352-7.

28. Kawaguchi Y, Hanaoka J, Ohshio Y, et al. A risk score to predict postoperative complications after lobectomy in elderly lung cancer patients. Gen Thorac Cardiovasc Surg 2018;66:537-42.

29. Hino H, Karasaki T, Yoshida Y, et al. Competing Risk Analysis in Lung Cancer Patients Over 80 Years Old Undergoing Surgery. World J Surg 2019;43:1857-66.

30. Agatston AS, Janowitz WR, Hildner FJ, et al. Quantification of coronary artery calcium using ultrafast computed tomography. J Am Coll Cardiol 1990;15:827-32.

31. Xie X, Zhao Y, De Bock GH, et al. Validation and prognosis of coronary artery calcium scoring in nontriggered thoracic computed tomography: Systematic review and meta-analysis. Circ Cardiovasc Imaging 2013;6:514-21.

32. Kim YK, Sung YM, Cho SH, et al. Reliability analysis of visual ranking of coronary artery calcification on low-dose CT of the thorax for lung cancer screening: comparison with ECG-gated calcium scoring CT. Int J Cardiovasc Imaging 2014;30:81-7.

33. Whitlock MC, Yeboah J, Burke GL, et al. Cancer and its association with the development of coronary artery calcification: An assessment from the multi-ethnic study of atherosclerosis. J Am Heart Assoc 2015;4:e002533.

34. Vinter N, Christesen AMS, Mortensen LS, et al. Relation of Coronary Artery Calcium Score and Risk of Cancer (from a Danish Population-Based Follow-up Study in Patients Who Underwent Cardiac Computed Tomography). Am J Cardiol 2017;120:542-9.

35. Ferguson MK, Saha-Chaudhuri P, Mitchell JD, et al. Prediction of major cardiovascular events after lung resection using a modified scoring system. Ann Thorac Surg 2014;97:1135-40.

36. Khuri SF, Daley J, Henderson W, et al. The Department of Veterans Affairs' NSQIP: The first national, validated, outcome-based, risk-adjusted, and peer-controlled program for the measurement and enhancement of the quality of surgical care. Ann Surg 1998;228:491-507.

37. Gaudio C, Tanzilli A, Mei M, et al. Concomitant screening of coronary artery disease and lung cancer with a new ultrafast-low-dose Computed Tomography protocol: A pilot randomised trial. Sci Rep 2019;9:13872.

38. Christensen JL, Sharma E, Gorvitovskaia AY, et al. Impact of slice thickness on the predictive value of lung cancer screening computed tomography in the evaluation of coronary artery calcification. J Am Heart Assoc 2019;8:e010110.
Cite this article as: Shipe ME, Maiga AW, Deppen SA, Edwards GC, Marmor HN, Pinkerman R, Smith GT, Lio E, Wright JL, Shah C, Nesbitt JC, Grogan EL. Preoperative coronary artery calcifications in veterans predict higher allcause mortality in early-stage lung cancer: a cohort study. J Thorac Dis 2021;13(3):1427-1433. doi: 10.21037/jtd-20-2102 